\title{
Factores predictores de la violencia relacional en la adolescencia
}

\author{
Predictive factors of relational violence in adolescence
}

\author{
Ana Romero-Abrio ${ }^{\mathrm{a}, *}$, Gonzalo Musitua Juan Evaristo Callejas-Jerónimo ${ }^{\mathrm{a}}$, \\ Juan Carlos Sánchez-Sosab, María Elena Villarreal-González \\ aUniversidad Pablo de Olavide, España \\ bUniversidad Autónoma de Nuevo León, México
}

\section{Resumen}

El objetivo del presente trabajo fue examinar la violencia relacional entre iguales desde una perspectiva psicosocial, analizando variables familiares (funcionamiento y comunicación familiar), sociales (reputación social - percibida e ideal - y actitud hacia la autoridad institucional) e individuales (malestar psicológico e ideación suicida) en adolescentes escolarizados. La muestra fue de 8115 adolescentes, con edades comprendidas entre los 11 y los 16 años, del estado de Nuevo León, México. Se realizó un análisis de regresión lineal múltiple, paso a paso, cuyos resultados mostraron que la dimensión más importante en la predicción de la conducta violenta relacional es la autopercepción no conformista, seguido del malestar psicológico. Finalmente, se discuten estos resultados y sus implicaciones.

Palabras clave: violencia relacional, reputación social, actitud hacia la autoridad, comunicación familiar, malestar psicológico.

\begin{abstract}
The aim of this research was to study relational violence between peers from a psychosocial perspective, by analyzing family variables (family functioning and communication), social variables (social reputation -both perceived and ideal- and attitude towards institutional authority), and individual variables (psychological distress and suicide ideation) in adolescent students. The sample consisted of 8,115 adolescents aged 11 to 16 years from the State of Nuevo León, Mexico. We performed a forward stepwise multiple regression analysis, the results of which showed that the most important factor in the prediction of violent relational behavior at school is the nonconformist self-perception, followed by psychological distress. Finally, these results and their implications are discussed.
\end{abstract}

Keywords: relational violence, social reputation, attitude towards institutional authority, family communication, psychological distress.
Para citar este artículo:

Romero-Abrio, A., Musitu, G., Callejos-Jerónimo, J., Sánchez-Sosa, J., \& Villarreal-González, M. (2018). Factores predictores de la violencia relacional en la adolescencia. Liberabit, 24(1), 29-43. doi: 10.24265/ liberabit.2018.v24n1.03
Este es un artículo Open Access bajo la licencia Creative Commons Atribución-NoComercial-CompartirIgual 4.0

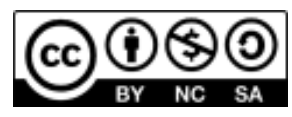




\section{Introducción}

La violencia escolar es un problema socioeducativo que perjudica gravemente el proceso de enseñanzaaprendizaje y las relaciones sociales en el aula entre compañeros y entre alumnos y profesores (Steffgen, Recchia, \& Viechtbauer, 2013). Diferentes estudios han puesto de relieve que la violencia escolar tiene consecuencias negativas en el funcionamiento y funciones de la escuela: por un lado, impacta en la motivación del profesorado hacia su trabajo, y, por otro, pone el foco de atención en los episodios violentos, por lo que se corre el riesgo de desatender tanto a los objetivos educativos del centro como al alumnado que no presenta estos problemas de conducta (Buelga, Martínez-Ferrer, \& Musitu, 2016; Cava \& Martínez, 2013; Trianes, 2000).

Han sido numerosas las investigaciones relacionadas con los efectos de la violencia, fundamentalmente en el alumnado, debido al incremento de la frecuencia y gravedad de este tipo de conductas en los centros educativos (Crespo-Ramos, Romero-Abrio, MartínezFerrer, \& Musitu, 2017; Debarbieux, 2006; Kawabata, 2016). En México, según la Encuesta Nacional de Salud y Nutrición (ENSANUT), la violencia en adolescentes de edades comprendidas entre los 10 y los 19 años se incrementó significativamente del 2006 (3.3\% en chicos y 1.3\% en chicas) al 2012 (4.4\% en chicos y 3.4\% en chicas), lo cual representa un incremento de más del 1.1\% y del 2.1\% respectivamente (Gutiérrez et al., 2013).

Probablemente, la clasificación de violencia más consensuada entre los investigadores sea la de Little, Brauner, Jones, Nock y Hawley (2003), en la que se hace referencia tanto a su forma como a sus funciones. Respecto de la forma, los autores distinguen entre violencia manifiesta, que alude a comportamientos que implican una confrontación directa hacia otros con la intención de causar daño (golpear, pegar u otros), y violencia relacional, cuyo objetivo es dañar la reputación social o el estatus social de las víctimas y aislarla de su grupo de amistades, utilizando incluso al grupo de compañeros (contando rumores, exclusión o rechazo social u otros). En cuanto a sus funciones, se distingue entre aquella que implica una conducta violenta sin que medie un estímulo desencadenante (violencia ofensiva); aquella que supone una respuesta defensiva ante alguna provocación (violencia reactiva) y, por último, la que conlleva un comportamiento deliberado de anticipación de beneficios y que está controlado por refuerzos externos (la acción instrumental) (Little et al., 2003).

La literatura científica ha constatado ampliamente las consecuencias negativas de la violencia manifiesta en el ajuste psicosocial del adolescente (Crespo-Ramos et al., 2017; Meeus, Van de Schoot, Hawk, Hale, \& Branje, 2016; Moreno, Ramos, Martínez, \& Musitu, 2010), sin embargo, los estudios de la violencia relacional, a pesar de que ésta tiene implicaciones igualmente perjudiciales, son aún escasos (Voulgaridou \& Kokkinos, 2015).

Respecto del sexo de los agresores, es de interés subrayar que en diversos trabajos se confirma que, ya desde la etapa de preescolar, los comportamientos agresivos directos de agresión física y verbal son más frecuentes entre los chicos (Card, Stucky, Sawalani, \& Little, 2008; Carrascosa, Cava, \& Buelga, 2015). Sin embargo, la evidencia empírica con respecto a la violencia relacional es ciertamente más controvertida. De esta manera, mientras que autores como Ettekal \& Ladd (2015) encuentran que las chicas participan más que los chicos en comportamientos de violencia relacional, otros autores no hallan diferencias entre ambos sexos (Card et al., 2008; Tseng, Banny, Kawabata, Crick, \& Gau, 2013). Como señalan Putallaz et al. (2007), pese a la falta de consenso, parece que están más acuerdo con la idea de que las chicas cuando se implican en comportamientos violentos tienden a utilizar más frecuentemente la violencia relacional que la violencia manifiesta. Este es un aspecto que, por su novedad e interés en la explicación de la violencia en función del sexo, merecería una mayor exploración. 
Con el objetivo de explicar tanto la violencia manifiesta como la relacional, los investigadores se han centrado en analizar su asociación con variables relacionadas con el ajuste psicosocial del adolescente. Respecto de variables familiares, se ha constatado que el funcionamiento, la comunicación y el clima familiar están íntimamente relacionados con la conducta violenta en la adolescencia (Estévez, Musitu, \& Herrero, 2005; Withers, McWey, \& Lucier-Greer, 2016). En relación a las variables escolares, se ha señalado que ciertos factores como el clima, los vínculos entre iguales y la calidad de la relación profesor-alumno tienen relación con las conductas disruptivas de los alumnos (Buelga et al., 2016; Villalta \& Saavedra, 2012). Asimismo, y con respecto a variables sociales, se ha observado que el deseo o motivación por conseguir una reputación o reconocimiento social, constituye un factor de riesgo respecto de la implicación en comportamientos antisociales y violentos en la adolescencia (Emler \& Reicher, 2005; Kawabata, Tseng, \& Crick, 2014). Este tipo de conductas podría entenderse como la respuesta ante la búsqueda de una determinada reputación social fundamentada en el respeto, el liderazgo, el poder en el grupo y el no-conformismo (Juvonen, Wang, \& Espinoza, 2013; Musitu, Estévez, \& Emler, 2007).

Una variable que es cada vez más considerada en los estudios sobre la violencia y el clima escolar en la adolescencia es la actitud hacia la autoridad institucional (Cava, Musitu, Buelga, \& Murgui, 2010; Moral \& Ovejero, 2013), puesto que se ha constatado que los adolescentes con problemas de conducta violenta tienden a presentar actitudes desfavorables hacia la autoridad institucional, tanto en el ámbito escolar- hacia la figura del profesorado- como en otros contextos sociales -hacia instituciones como la policía (Emler, 2008; Emler \& Reicher, 2005).

En diversas investigaciones se ha demostrado que tanto las víctimas de violencia manifiesta como relacional presentan síntomas depresivos, problemas de ansiedad, malestar psicológico, así como ideación suicida (Espelage \& Holt, 2013; Sanchez-Sosa, Villarreal-Gonzalez, \& Musitu, 2013). Del mismo modo, una mayor percepción y exposición a la violencia en la adolescencia puede afectar el estado emocional de los sujetos, llevándolos a presentar una sintomatología ansiosa o depresiva, síntomas que se asocian al uso de sustancias (Pérez-Islas, DíazNegrete, \& Fernández-Caceres, 2014). Teniendo en cuenta estos precedentes, consideramos que la violencia relacional no ha tenido el mismo interés en la comunidad científica que la violencia manifiesta $y$, en consecuencia, creemos que esta laguna en el conocimiento respecto de la violencia relacional entre iguales merece ser analizada con mayor exhaustividad. Analizando los antecedentes previos y considerando que son todavía escasos los trabajos que examinen cómo se define la violencia relacional en la escuela incorporando variables psicosociales (Moreno, Estévez, Murgui, \& Musitu, 2009; Tseng et al., 2013), el objetivo del presente trabajo es estudiar el poder predictivo de diferentes variables psicosociales en la violencia relacional en la adolescencia, tales como la reputación social, el funcionamiento y la comunicación familiar, la actitud hacia la autoridad institucional, el malestar psicológico y la ideación suicida.

\section{Método}

El diseño de este estudio fue ex postfacto explicativo y transversal.

\section{Participantes}

La selección de los participantes se realizó mediante un muestreo estratificado proporcional en función de centros educativos, urbanos y suburbanos, que constituían un universo de 984 centros del Estado de Nuevo León (México). La muestra seleccionada fue de 118 centros educativos (62 urbanos y 56 suburbanos), con un total de 8115 adolescentes ( $51.5 \%$ chicos y $48.5 \%$ chicas), de los cuales $62.3 \%$ pertenecían a escuelas urbanas y $37.7 \%$ a escuelas suburbanas. Las edades estaban comprendidas entre los 11-13 años (54\%) y los 14-16 años (46\%). En 
relación con el grado escolar, 35.5\% cursaban primer grado, 33.8\% cursaban segundo grado y 30.7\% cursaban tercer grado. En este trabajo los valores perdidos por escalas o subescalas, siempre que no superaran el $15 \%$, se trataron mediante el método de imputación por regresión (Little \& Rubin, 2002). La detección de valores atípicos univariantes se realizó mediante la exploración de puntuaciones estandarizadas, siguiendo los criterios indicados por Hair, Anderson, Tatham y Black (1999).

\section{Instrumentos}

Escala de Conducta Violenta Relacional (Little, Henrich, Jones, \& Hawley, 2003). Se ha utilizado la subescala de violencia relacional, la cual consta de 12 ítems tipo Likert con cuatro opciones de respuesta (1 = nunca, 4 = siempre) (e.g., «Soy una persona que trata con indiferencia a los demás»). El alfa de Cronbach fue de .76. El análisis factorial confirmatorio (CFA) mostró un aceptable ajuste a los datos [SB $\chi^{2}$ $=191.8766, g l=24, p<.001, \mathrm{CFI}=.956$, RMSEA $=.029(.026, .033)]$.

Escala de Reputación Social (Carroll, Baglioni, Houghton, \& Bramston, 1999). Consta de 15 ítems con cuatro opciones de respuesta ( $1=$ nunca, $4=$ siempre), que miden las siguientes dimensiones: autopercepción no-conformista (e.g., "Soy un/a chico/a rebelde»), autopercepción conformista (e.g., «Me llevo bien con los demás»), autopercepción de la reputación (e.g., "Tengo buena fama»), self público ideal no-conformista (e.g., «Me gustaría que los demás pensasen que soy un chico/a rebelde»), self público ideal conformista (e.g., «Me gustaría que los demás pensasen que me llevo bien con los demás») y self público ideal reputacional (e.g., «Me gustaría que los demás pensasen que tengo buena fama»). El alfa de Cronbach fue .85, .75, .76, .78, .72 y .71, respectivamente. El CFA mostró buen ajuste del modelo a los datos $\left[\mathrm{SB} \chi^{2}=979.6105, \mathrm{gl}=53, \mathrm{p}<\right.$ $.001, \mathrm{CFI}=.935$, RMSEA $=.046(.044, .049)]$ para reputación real, y $\left[\mathrm{SB} \chi^{2}=702.055, g l=55, p<.001\right.$,
CFI $=.950$, RMSEA $=.038(.036, .041)]$ para reputación ideal.

Escala de Funcionamiento Familiar (APGAR) (Smilkstein, Ashworth, \& Montano, 1982) Evalúa la cohesión y la adaptabilidad del funcionamiento familiar (e.g., «Estás satisfecho/a con el tiempo que tu familia y tú pasáis juntos»). Consta de cinco ítems con tres opciones de respuesta ( $0=$ casi nunca, $1=$ a veces $\mathrm{y}$ 2 = casi siempre). El alfa de Cronbach fue de .80. El CFA mostró buen ajuste del modelo a los datos [SB $\chi^{2}$ $=40.41, g l=4, p<.001$, CFI $=.996$, RMSEA $=$ $.033(.025, .043)]$.

Escala de Comunicación Padres-Hijos (PACS) (Barnes \& Olson, 1982). Esta escala tipo Likert consta de dos subescalas de 20 ítems, comunicación con la madre y comunicación con el padre, con cinco opciones de respuesta ( 1 = nunca, 5 = siempre). Cada subescala consta de dos dimensiones: comunicación abierta (e.g., «Me presta atención cuando le hablo») y comunicación ofensiva (e.g., «Intenta ofenderme cuando se enfada conmigo»). El alfa de Cronbach fue de .89 (padre) y .88 (madre) en comunicación abierta; y de .64 (padre) y .69 (madre) en comunicación ofensiva. El CFA mostró buen ajuste del modelo a los datos $\left[\mathrm{SB} \chi^{2}=2602.98, g l=128, p<.001\right.$, CFI $=.953$, RMSEA $=.049(.047, .050)]$.

Escala de Actitudes hacia la Autoridad Institucional en Adolescentes (AAI-A) (Cava, Estévez, Buelga, \& Musitu, 2013). Consta de 10 ítems con cuatro opciones de respuesta ( 1 = nada de acuerdo, 4 = totalmente de acuerdo) que miden dos factores: actitud positiva hacia la autoridad (e.g., «Los profesores son justos a la hora de evaluar») y actitud positiva hacia la transgresión de normas (e.g., «Si una regla escolar no te gusta, lo mejor es saltársela»). El alfa de Cronbach fue de .90 y .92, respectivamente. El CFA mostró un buen ajuste a los datos $\left[\mathrm{SB} \chi^{2}=318.42, g l=23, p<.001\right.$, CFI $=.976$, RMSEA $=.040(.036, .044)]$. 
Escala de Malestar Psicológico (K-10) (Kessler \& Mroczek, 1994). La escala consta de 10 ítems tipo Likert con cinco opciones de respuesta ( 1 = nunca, 5 = siempre) que evalúan síntomas depresivos y de ansiedad (e.g., «Con qué frecuencia te has sentido inquieto/a o intranquilo/a»). El alfa de Cronbach fue de .90. El CFA mostró un buen ajuste a los datos $\left[\mathrm{SB} \chi^{2}=512.36, g l=29, p<.001\right.$, CFI $=.981$, RMSEA $=.045(.042, .049)]$.

Escala de Ideación Suicida (Roberts, 1980). Fue empleada la versión adaptada de Mariño, Chaparro, \& González (1993). Evalúa la frecuencia de pensamientos suicidas en la última semana (e.g., «Tenía pensamientos sobre la muerte») y consta de cuatro preguntas tipo Likert con cuatro opciones de respuesta ( $1=0$ días, $4=5-7$ días $)$. El alfa de Cronbach fue de .84. El CFA presentó un buen ajuste a los datos $\left[\mathrm{SB} \chi^{2}=1.643, g l=1, p=.199, \mathrm{CFI}=\right.$ .991 , RMSEA $=.009(.000, .032)]$.

\section{Procedimiento}

La planificación y el desarrollo del proyecto se realizó entre la Universidad Autónoma de Nuevo León (UANL) y la Secretaria de Educación (SE). Una vez informados y concedidos los permisos, se administraron los instrumentos en los centros escolares seleccionados, previo permiso de los padres y del alumnado. En aquellas aulas en las que había alumnado con problemas de lectura y comprensión, se les administró el instrumento de forma individualizada y se les ofreció apoyo. La participación fue voluntaria y anónima, con un total de 19 alumnos (21\%) que rechazaron participar en el proyecto.

\section{Análisis de datos}

En primer lugar, se realizó un análisis de clúster en dos fases (análisis bietápico y k-means) para la variable violencia relacional (VR); y se obtuvieron tres grupos (baja, moderada y alta VR). En segundo lugar, se efectuó un análisis discriminante con las dimensiones objeto de estudio, con la finalidad de obtener aquellas que más discriminaban entre los clústeres de alta y baja VR. Esta técnica es especialmente adecuada en muestras amplias (Closas, Arriola, Kuc, Amarilla, \& Jovanovich, 2013), como la del presente trabajo. En los posteriores análisis se incluyeron únicamente las dimensiones con saturación $>.30$.

Posteriormente, se realizó un análisis correlacional. Por último, se calculó una regresión lineal múltiple por pasos, con el objetivo de analizar la capacidad predictiva en la variable criterio (VR) de las variables objeto de estudio (funcionamiento familiar, comunicación ofensiva en padre, comunicación ofensiva en madre, malestar psicológico, ideación suicida, actitud positiva hacia la autoridad institucional, actitud positiva hacia la transgresión de normas sociales, autopercepción no-conformista y self público ideal no-conformista). Se utilizó el software SPSS edición 20.

\section{Resultados}

Los resultados de la muestra en su conjunto confirman que la VR correlaciona significativamente con todas las variables consideradas en este trabajo (Tabla 1). 


\section{Tabla 1}

Análisis correlacional

\begin{tabular}{|c|c|c|c|c|c|c|c|c|c|c|}
\hline Variables & 1 & 2 & 3 & 4 & 5 & 6 & 7 & 8 & 9 & 10 \\
\hline 1. $\quad$ Violencia relacional & - & & & & & & & & & \\
\hline 2. Funcionamiento familiar & $-.12^{* *}$ & - & & & & & & & & \\
\hline 3. Ideación suicida & $.18^{* *}$ & $-.26^{* *}$ & - & & & & & & & \\
\hline 4. Malestar psicológico & $.24^{* *}$ & $-.14^{* *}$ & $.49^{* *}$ & - & & & & & & \\
\hline 5. Actitud positiva autoridad institucional & $-.20^{* *}$ & $.31^{* *}$ & $-.13^{* *}$ & $-.09^{* *}$ & - & & & & & \\
\hline 6. Actitud positiva transgresión normas sociales & $.23^{* *}$ & $-.13^{* *}$ & $.16^{* *}$ & $.13^{* *}$ & $-.11^{* *}$ & - & & & & \\
\hline 7. Autopercepción no-conformista & $.31^{* *}$ & $-.16^{* *}$ & $.24^{* *}$ & $.26^{* *}$ & $-.25^{* *}$ & $.42^{* *}$ & - & & & \\
\hline 8. Self público ideal no-conformista & $.23^{* *}$ & $-.16^{* *}$ & $.18^{* *}$ & $.12^{* *}$ & $-.20^{* *}$ & $.35^{* *}$ & $.55^{* *}$ & - & & \\
\hline 9. Comunicación ofensiva con la madre & $.20^{* *}$ & $-.09^{* *}$ & $.26^{* *}$ & $.34^{* *}$ & $-.09^{* *}$ & $.18^{* *}$ & $.25^{* *}$ & $.16^{* *}$ & - & \\
\hline 10. Comunicación ofensiva con el padre & $.14^{* *}$ & -.02 & $.16^{* *}$ & $.25^{* *}$ & $-.05^{* *}$ & $.14^{* *}$ & $.19^{* *}$ & $.13^{* *}$ & $.69^{* *}$ & - \\
\hline
\end{tabular}

Nota: ${ }^{* *} p<.01$ (bilateral); ${ }^{* * *} p<.001$ (bilateral).

El análisis de regresión lineal múltiple, paso a paso, cuyos resultados se presentan en la Tabla 2, mostró que, de las variables predictoras de la conducta violenta relacional en la escuela, seis resultaron estadísticamente significativas: la autopercepción no conformista ( $\beta=.147)$, el malestar psicológico $(\beta=$ .141 ), la actitud positiva hacia la autoridad institucional ( $\beta=-.115$ ), la actitud positiva hacia la transgresión de normas sociales $(\beta=.104)$, la comunicación problemática con la madre $(\beta=.077)$ y el self público ideal no conformista $(\beta=.061)$. Los coeficientes de regresión estandarizados $\beta$ indican que todas las variables tienen cierto peso sobre la variable criterio. De acuerdo con esta afirmación, los porcentajes de varianza explicada (coeficientes de determinación ajustados) por cada una de tales variables predictoras fueron de magnitud media para las seis variables, respectivamente: $9.6 \%, 12.2 \%, 13.5 \%, 14.7 \%, 15.3 \%$ y $15.5 \%$. Alta autopercepción no conformista, alto malestar psicológico, bajas actitudes positivas hacia la autoridad institucional, altas actitudes positivas hacia la transgresión de normas sociales, alta comunicación problemática con la madre y alto self público ideal no conformista resultaron variables predictoras de la conducta violenta relacional en la escuela, siendo su poder explicativo medio, ya que explican el $15.5 \%$ de la varianza.

La interpretación del $\beta$ de cada variable predictora sobre la variable criterio, la violenta relacional, por ejemplo, sería la siguiente: por cada unidad sumada a la variable autopercepción no conformista se incrementa .147 puntos la conducta violenta relacional en la escuela. 
Tabla 2

Análisis de regresión múltiple por pasos para variables predictoras de violencia relacional para el conjunto de la muestra

\begin{tabular}{|c|c|c|c|c|c|c|}
\hline & no & $\begin{array}{l}\text { Coeficientes } \\
\text { estandarizados }\end{array}$ & $\begin{array}{c}\text { Coeficientes } \\
\text { estandarizados }\end{array}$ & $t$ & $p$ & \\
\hline Factores & B & Tip. Error & $\beta$ & & & \\
\hline 1 (Constante) & .98 & .011 & & 91.94 & .000 & $\mathrm{R}^{2}=.095$ \\
\hline ANC & .21 & .01 & .31 & 29.28 & .000 & $F=857.18$ \\
\hline 2 (Constante) & .89 & .01 & & 74.79 & .000 & $\mathrm{R}^{2}=.122$ \\
\hline ANC & .18 & .01 & .26 & 24.56 & .000 & $F=561.95$ \\
\hline MPS & .06 & .004 & .17 & 15.53 & .000 & \\
\hline 3 (Constante) & 1.07 & .02 & & 53.88 & .000 & $\mathrm{R}^{2}=.135$ \\
\hline ANC & .16 & .007 & .23 & 21.33 & .000 & $F=423.62$ \\
\hline MPS & .06 & .004 & .16 & 15.33 & .000 & \\
\hline APAI & -.05 & .005 & -.12 & -11.37 & .000 & \\
\hline 4 (Constante) & 1.03 & .02 & & 51.13 & .000 & $\mathrm{R}^{2}=.147$ \\
\hline ANC & .12 & .01 & .18 & 15.58 & .000 & $F=350.29$ \\
\hline MPS & .06 & .004 & .16 & 15.22 & .000 & \\
\hline APAI & -.05 & .005 & -.12 & -11.36 & .000 & \\
\hline APTNS & .06 & .005 & .12 & 10.61 & .000 & \\
\hline 5 (Constante) & .99 & .02 & & 48.09 & .000 & $\mathrm{R}^{2}=.153$ \\
\hline ANC & .12 & .01 & .18 & 14.69 & .000 & $F=291.87$ \\
\hline MPS & .05 & .004 & .14 & 12.49 & .000 & \\
\hline APAI & -.05 & .005 & -.12 & -11.26 & .000 & \\
\hline APTNS & .05 & .005 & .11 & 9.98 & .000 & \\
\hline COM & .03 & .005 & .08 & 7.07 & .000 & \\
\hline 6 (Constante) & .97 & .02 & & 45.52 & .000 & $\mathrm{R}^{2}=.155$ \\
\hline ANC & .10 & .01 & .15 & 11.02 & .000 & $F=248.01$ \\
\hline MPS & .05 & .004 & .14 & 12.69 & .000 & \\
\hline APAI & -.05 & .005 & -.115 & 10.87 & .000 & \\
\hline APTNS & .05 & .005 & .10 & 9.07 & .000 & \\
\hline COM & .03 & .005 & .08 & 6.92 & .000 & \\
\hline SPINC & .04 & .01 & .06 & 4.94 & .000 & \\
\hline \multirow{7}{*}{$\begin{array}{l}7 \text { (Constante) } \\
\text { ANC } \\
\text { MPS } \\
\text { APAI } \\
\text { APTNS } \\
\text { COM } \\
\text { SPINC }\end{array}$} & .96 & .02 & & 45.03 & .000 & $\mathrm{R}^{2}=.157$ \\
\hline & .10 & .01 & .15 & 11.29 & .000 & $F=216.20$ \\
\hline & .05 & .004 & .14 & 12.93 & .000 & \\
\hline & -.05 & .005 & -.12 & -11.16 & .000 & \\
\hline & .05 & .005 & .10 & 9.14 & .000 & \\
\hline & .03 & .005 & .08 & 6.87 & .000 & \\
\hline & .04 & .01 & .06 & 4.77 & .000 & \\
\hline
\end{tabular}

Nota: $p$ <.001; VR: variable criterio; ANC: autopercepción no conformista; MPS: malestar psicológico; APAI: actitud positiva hacia autoridad institucional; APTNS: actitud positiva hacia transgresión de normas sociales; COM: comunicación ofensiva madre; SPINC: Self público ideal no conformista. 


\section{Discusión}

En el presente trabajo de investigación, el objetivo fue examinar los factores psicosociales que intervienen en el proceso de construcción de la conducta violenta relacional en la adolescencia. De los determinantes de la violencia relacional mediante el análisis de regresión, los resultados confirman parcialmente las relaciones esperadas (poder explicativo medio, es decir, que un $15.5 \%$ de la justificación de la violencia relacional es explicado por este conjunto de variables seleccionadas).

Así se muestra que el principal factor predictivo de la violencia relacional en la escuela es la autopercepción no conformista, dimensión de la reputación social. En otros términos, los adolescentes que poseen una alta autopercepción no conformista muestran una mayor violencia relacional. Este resultado es interesante y permite subrayar la importancia de incorporar nuevas variables para explicar la violencia escolar, como es el caso de la reputación social, que en el ámbito de la violencia relacional ha sido muy poco explorada. En este sentido, los resultados suscriben los obtenidos por los autores Estévez, Jiménez y Cava (2016), en los que se subraya que, en la adolescencia, la reputación del agresor y de la víctima, así como su pertenencia o no a determinadas categorías o grupos sociales, influyen en la conducta violenta entre iguales. Ello nos remite a la idea de que para el adolescente la popularidad, el liderazgo y el poder entre los pares constituye un aspecto central de su vida (Gangel, Keane, Calkins, Shanahan, \& O’Brien, 2017) . El malestar psicológico en los adolescentes contribuye de manera significativa en la explicación de la conducta violenta relacional. El malestar psicológico junto con la sintomatología depresiva también se ha considerado un factor determinante de la conducta violenta (Kushner, Herzhoff, Vrshek-Schallhorn, \& Tackett, 2017; Villarreal-González, Sánchez-Sosa, Veiga, \& Moral-Arroyo, 2011). En relación con estos hallazgos, se confirma lo que en algunos estudios se ha observado con respecto a la violencia escolar
(Villarreal-González et al., 2011) en el sentido de que tanto los agresores como las víctimas presentan más desórdenes psicológicos que el resto de adolescentes. No obstante, en otros trabajos no se ha observado esta relación entre la violencia escolar y la presencia de sintomatología depresiva y estrés (Kawabata, 2016). Finalmente, en otros estudios, se observó una coocurrencia sumamente pequeña (alrededor del 58\%) entre violencia y depresión (Garnefski \& Diekstra, 1997). Estos datos sugieren que el comportamiento agresivo no tiene que ser ni consecuencia ni origen directo de la depresión y estrés (Estévez, Musitu, \& Herrero, 2005). Muy al contrario, en trabajos relativamente recientes, se ha constatado que la mayoría de los agresores presentan un adecuado ajuste emocional (Brendgen, Vitaro, Turgeon, Poulin, \& Wanner, 2004), posiblemente, debido a su también adecuado ajuste social en la escuela y al apoyo que reciben de sus amigos, factores que generan un déficit de desarrollar problemas de carácter depresivo. Creemos que estas dimensiones merecen de una mayor exploración en el ámbito específico de la violencia relacional por su interés tanto científico como en el ámbito de intervención.

Como se infiere también de los resultados, los adolescentes que presentan baja actitud positiva hacia la autoridad institucional y alta actitud positiva hacia la transgresión de normas sociales muestran una mayor violencia relacional en el contexto escolar. Del mismo modo, se ha observado que el self público ideal no conformista, dimensión de la reputación social, contribuye de forma significativa en la conducta violenta relacional. La conexión entre la reputación social y la actitud hacia la autoridad institucional parecen que se fundamentan en que, para algunos adolescentes, la reputación se consigue a través de las actitudes que el adolescente tiene hacia la transgresión de normas que son recompensadas en términos de estatus social entre sus compañeros (Gini, 2006). En este sentido, algunos comportamientos transgresores como conductas violentas en el medio escolar (Putallaz et al., 2007; Martínez-Ferrer, Murgui, Musitu, \& Monreal-Gimeno, 2008), 
conductas delictivas (Buelga \& Musitu, 2006; Emler \& Reicher, 2005), consumo de drogas (Buelga, Ravenna, Musitu, \& Lila, 2006) y conductas disruptivas en el aula (Luthar \& Ansary, 2005) incitan a pensar que estos comportamientos permiten a algunos adolescentes su reconocimiento social.

También se ha observado en numerosos trabajos que algunos agresores escolares son percibidos por sus iguales como figuras importantes en su grupo de iguales (Hawley \& Vaughn, 2003; Vaillancourt, 2001) y son populares y aceptados entre sus compañeros (Gangel et al., 2017). Estos resultados son coherentes con la idea de que el comportamiento violento del adolescente agresor se vincula a ciertas necesidades de protagonismo y de popularidad (Cillessen, Mayeux, Ha, Bruyn, \& Lafontana, 2014; Gangel et al., 2017). Por lo tanto, a la luz de estos datos, este tipo de violencia relacional entre iguales parece que se explica más por la insatisfacción personal con respecto al estatus social que tiene el adolescente (reputación ideal) que por la reputación percibida. En efecto, Cillessen et al. (2014) sugiere que, los adolescentes tienen popularidad y reconocimiento social entre sus compañeros con conductas que no son transgresoras (por ejemplo, en el deporte). Esto explicaría que una alta reputación percibida no necesariamente es un factor de riesgo de la conducta violenta, pero sí lo es el deseo de tener una mayor reputación entre los pares. De hecho, los resultados del presente trabajo corroboran la idea de que existe una relación determinante entre la reputación social del adolescente y la conducta violenta, y como se confirma en el trabajo de Buelga et al. (2008), se observó una confrontación directa física y verbal entre iguales adolescentes. Por lo tanto, como se apunta en este trabajo y en el de Juvonen et al. (2013), parece que efectivamente la reputación social del adolescente es una variable relevante para explicar, además de la conducta delictiva, la implicación del adolescente en comportamientos violentos tanto manifiestos como relacionales.
Un hallazgo que resulta relevante en este estudio es el de la comunicación con los progenitores. La comunicación ofensiva con el padre no parece influir significativamente en la conducta violenta relacional; sin embargo, los adolescentes que informan de una comunicación problemática con la madre sí presentan una mayor incidencia en violencia relacional. Este resultado está en la línea de los obtenidos en anteriores investigaciones respecto de la no violencia, en los que se destaca el fuerte vínculo existente entre la relación padre-adolescente que, cuando es positiva y abierta, el ajuste psicosocial del hijo es alto (Aymerich, 2015; Welsh, Buchanan, Flouri, \& Lewis, 2004). También se ha observado que un buen funcionamiento familiar, cohesión, apoyo, confianza e intimidad favorece el ajuste conductual y psicológico de los hijos (Moral \& Ovejero, 2013; Musitu \& García, 2004), e inversamente, un déficit en estas dimensiones constituye uno de los factores de riesgo más directamente relacionados con los problemas de conducta violenta o delictiva en niños y adolescentes (Buelga et al., 2016). Con respecto a la diferenciación entre la comunicación con la madre y con el padre, los resultados obtenidos apoyan la idea de que aún hoy en países como España y México, la madre sigue siendo el principal activo del funcionamiento familiar y sobre la que gira la construcción de la identidad de los hijos (Fuentes, Motrico, \& Bersabé, 2003). También se ha constatado en recientes estudios (Estévez, Emler, Cava, \& Inglés, 2014; Sánchez-Sosa, Villarreal-González, Ávila, Jiménez, \& Musitu, 2014; Varela, Ávila, \& Martínez, 2013) una mayor influencia de la figura del padre en la conducta violenta de los adolescentes cuando las relaciones no son positivas. Del mismo modo, mientras la relación con el padre es relevante tanto para chicos como para chicas, la calidad de la relación con la madre, y, específicamente, la existencia de una comunicación positiva entre madre e hija, parece disminuir la implicación de las chicas en la violencia relacional (Carrascosa, Cava, \& Buelga, 2015).

Teniendo en cuenta los resultados obtenidos, consideramos que es imprescindible que tanto los 
padres y madres de familia como el profesorado dispongan de los recursos necesarios para poder detectar a tiempo cuándo los adolescentes están siendo víctimas de este tipo de violencia. Al tratarse de una conducta difícilmente observable y que ocurre de forma encubierta, requiere, para su prevención, de una sólida formación profesional, una gran sensibilidad y una indispensable colaboración entre la familia y la escuela.

Esta investigación tiene ciertas limitaciones. Por un lado, su carácter transversal no permite que se puedan establecer relaciones causales entre las variables analizadas, por lo que consideramos que sería interesante profundizar en el estudio de la violencia relacional utilizando estudios longitudinales. Por otro lado, la utilización de auto-informes puede proporcionar repuestas sesgadas de los sujetos, por lo que sería más enriquecedor para la investigación poder disponer de diferentes informantes, como padres/madres y profesorado, que puedan dar información más completa del comportamiento violento de los adolescentes. Pese a estas limitaciones, los resultados del presente estudio permiten progresar en un tema que continúa generando preocupación social, como es la violencia escolar, poniendo el foco de atención en la vertiente relacional o indirecta de la conducta violenta. Las implicaciones prácticas que se derivan de este trabajo van en la dirección de incorporar la violencia relacional en los programas de intervención en las escuelas, así como incluirla en la formación del profesorado y de los padres y madres, contribuyendo a una relación fluida entre la familia y la escuela.

\section{Conflicto de intereses}

Los autores firmantes declaran que no existe ningún conflicto de intereses relacionado con el artículo.

\section{Responsabilidad ética}

Los autores declaran que el estudio cumplió los valores éticos requeridos en una investigación con seres humanos, respetando los principios fundamentales incluidos en la Declaración Helsinki, en sus actualizaciones y en las normativas vigentes (consentimiento informado, derecho a la información, protección de datos personales, garantías de confidencialidad, no discriminación y gratuidad) con la posibilidad de abandonar el estudio en cualquiera de sus fases.

\section{Agradecimiento}

Este estudio ha sido realizado con el apoyo económico del proyecto de investigación «El acoso escolar en la adolescencia: variables individuales y familiares», en el marco del Programa de Apoyo a la Investigación Científica y Tecnológica (PAYCIT) de la Universidad Autónoma de Nuevo León (UANL) (México). 


\section{Referencias}

Aymerich, M. M. (2015). Celos y violencia en alumnos de enseñanza secundaria. Influencia de los estilos educativos familiares. (Tesis de doctorado). Recuperada de https:/www.tdx.cat/bitstream/handle/ 10803/352467/mmaymerich.pdf?sequence=1

Barnes, H., \& Olson, D. (1982). Parent adolescent communication scale. In D. H. Olson (Ed.), Family Inventories (pp. 145-182). SSt. Paul: Family Social Sciences, University of Minnesota.

Brendgen, M., Vitaro, F., Turgeon, L., Poulin, F., \& Wanner, B. (2004). Is there a dark side of positive illusions? Overestimation of social competence and subsequent adjustment in aggressive and nonaggressive children. Journal of Abnormal Child Psychology, 32(3), 305-320. doi: 10.1023/ B:JACP.0000026144.08470.cd

Buelga, S. \& Musitu, G. (2006). Famille et adolescence: Prévention de conduites à risque. En M. Zabalia \& D. Jacquet (Eds.), Adolescences d'aujourd'hui (pp.1735). Rennes: Presses Universitaires de France

Buelga, S., Martínez-Ferrer, B., \& Musitu, G. (2016). Family Relationships and Cyberbullying. In R. Navarro, / S. Yubero, \& E. Larrañaga (Eds.). Cyberbullying Across the Globe: Gender, Family, and Mental Health (pp. 99-114). España: Springer.

Buelga, S., Musitu, G., Murgui, S., \& Pons, J. (2008). Reputation, loneliness, satisfaction with life and aggressive behavior in adolescence. The Spanish Journal of Psychology, 11(1), 192-200. doi: $10.1017 / \mathrm{S} 1138741600004236$

Buelga, S., Ravenna, M., Musitu, G., \& Lila, M. (2006). Epidemiology and psychological risk factors associated with adolescent drug consumption. In J, Sandy \& G. Luc (Eds.) Handbook of Adolescent Development (pp. 337-385) New York: Psychology Press

Card, N. A., Stucky, B. D., Sawalani, G. M., \& Little, T. D. (2008). Direct and indirect aggression during childhood and adolescence: A meta-analytic review of gender differences, intercorrelations, and relations to maladjustment. Child Development, 79(5), 11851229. doi: 10.1111/j.1467-8624.2008.01184.x
Carrascosa, L., Cava, M. J., \& Buelga, S. (2015). Actitudes hacia la autoridad y violencia entre adolescentes: diferencias en función del sexo. Suma Psicológica, 22(2), 102-109. doi: 10.1016/j.sumpsi.2015.08.003

Carroll, A., Baglioni, A. J., Houghton, S., \& Bramston, P. (1999). At-risk and not at-risk primary school children: an examination of goal orientations and social reputations. The British Journal of Educational Psychology, 69(3), 377-92. doi: 10.1348/000709999157789

Cava, M. J., Estévez, E., Buelga, S., \& Musitu, G. (2013). Propiedades psicométricas de la escala de actitudes hacia la autoridad institucional en adolescentes (AAIA). Anales de Psicologia, 29(2), 540-548. doi: 10.6018/analesps.29.2.138031

Cava, M. J., \& Martínez, B. (2013). Adolescencia y familia:nuevos retos del siglo XXI. Mexico: Trillas.

Cava, M. J., Musitu, G., Buelga, S., \& Murgui, S. (2010). The relationships of family and classroom environments with peer relational victimization: an analysis of their gender differences. The Spanish Journal of Psychology, 13(1), 156-65. doi: 10.1017/S1138741600003747

Cillessen, A. H. N., Mayeux, L., Ha, T., de Bruyn, E. H., \& Lafontana, K. M. (2014). Aggressive effects of prioritizing popularity in early adolescence. Aggressive Behavior, 40(3), 204-213. doi: 10.1002/ ab. 21518

Closas, A. H., Arriola, E. A., Kuc, C. I., Amarilla, R., \& Jovanovich, E. C. (2013). Análisis multivariante, conceptos y aplicaciones en Psicología Educativa y Psicometría. Enfoques, 25(1), 65-92. Recuperado de http://www.scielo.org.ar/pdf/enfoques/v25n1/ v25n1a05.pdf

Crespo-Ramos, S., Romero-Abrio, A., Martínez-Ferrer, B., \& Musitu, G. (2017). Variables psicosociales y violencia escolar en la adolescencia. Psychosocial Intervention, 26(2), 125-130. doi: 10.1016/j.psi. 2017.05.002

Debarbieux, E. (2006). Violence à l'école: un défi mondial?. Paris: Armand Colin. 
Emler, N. P. (2008). Delinquents as a minority group: Accidental tourists in forbidden territory or voluntary émigrés? In F. Butera \& J. Levine (Eds.), Coping with minority status: Responses to exclusion and inclusion. Cambridge: Cambridge.

Emler, N., \& Reicher, S. (2005). Delinquency: Cause or consequence of social exclusion? In D. Abrams, M. Hogg, \& J. Marques (Eds.). The social psychology of inclusion and exclusion (pp. 211-241). New York, NY, US: Psychology Press.

Espelage, D. L., \& Holt, M. K. (2013). Suicidal Ideation and School Bullying Experiences After Controlling for Depression and Delinquency. Journal of Adolescent Health, 53(1), S27-S31. doi: 10.1016/ j.jadohealth.2012.09.017

Estévez, E., Emler, N. P., Cava, M. J., \& Inglés, C. J. (2014). Psychosocial adjustment in aggressive popular and aggressive rejected adolescents at school. Psychosocial Intervention, 23(1), 57-67. doi: 10.5093/in2014a6

Estévez, E., Jiménez, T. I., \& Cava, M. J. (2016). A CrossCultural Study in Spain and Mexico on School Aggression in Adolescence: Examining the Role of Individual, Family, and School Variables. CrossCultural Research, 50(2), 123-153. doi: 10.1177/ 1069397115625637

Estévez, E., Musitu, G., \& Herrero, J. (2005). El rol de la comunicación familiar y del ajuste escolar en la salud mental del adolescente. Salud Mental, 28(4), 81-89. Recuperado de http://www.uv.es/lisis/estevez/ estevez_salud

Estévez, E., Musitu, G., \& Herrero, J. (2005). The influence of violent behavior and victimization at school on psychological distress: the role of parents and teachers. Adolescence, 40(157), 183-196.

Ettekal, I., \& Ladd, G. W. (2015). Costs and benefits of children's physical and relational aggression trajectories on peer rejection, acceptance, and friendships: Variations by aggression subtypes, gender, and age. Developmental Psychology, 51(12), 1756-1770. doi: 10.1037/dev0000057
Fuentes, M. J., Motrico, E., \& Bersabé, R. (2003). Estrategias de socialización de los padres y conflictos entre padres e hijos en la adolescencia. Anuario de psicología/The UB Journal of Psychology, 34(3), 385-400.

Gangel, M. J., Keane, S. P., Calkins, S. D., Shanahan, L., \& O’Brien, M. (2017). The Association Between Relational Aggression and Perceived Popularity in Early Adolescence: A Test of Competing Hypotheses. The Journal of Early Adolescence, 37(8), 10781092. doi: 10.1177/0272431616642327

Garnefski, N., \& Diekstra, R. F. W. (1997). «Comorbidity» of behavioral, emotional, and cognitive problems in adolescence. Journal of Youth and Adolescence, 26(3), 321-338. doi: 10.1007/s10964005-0005-3

Gini, G. (2006). Bullying as a social process: The role of group membership in students' perception of intergroup aggression at school. Journal of School Psychology, 44(1), 51-65. doi: 10.1016/j.jsp.2005 .12 .002

Gutiérrez, J. P., Rivera-Dommarco, J. A., Shamah-Levy, T., Villalpando-Hernández, S., Franco, A., CuevasNasu, L., ... Hernández-Ávila, M. (2013). Encuesta Nacional de Salud y Nutrición 2012. Resultados Nacionales. 2a. ed. [National Survey of Health and Nutrition. National results]. Instituto Nacional de Salud Publica. Recuperado de http://ensanut.insp.mx/ informes/ENSANUT2012ResultadosNacionales 2Ed.pdf

Hair, J., Anderson, R., Tatham, R., \& Black, W. (1999). Análisis multivariante [Multivariate analysis]. España: Prentice Hall.

Hawley, P. H., \& Vaughn, B. E. (2003). Aggression and Adaptive Functioning: The Bright Side to Bad Behavior. Merrill-Palmer Quarterly, 49(3), 239242. doi: 10.1353/mpq.2003.0012

Juvonen, J., Wang, Y., \& Espinoza, G. (2013). Physical Aggression, Spreading of Rumors, and Social Prominence in Early Adolescence: Reciprocal Effects Supporting Gender Similarities? Journal of Youth and Adolescence, 42(12), 1801-1810. doi: 10.1007/ s10964-012-9894-0 
Kawabata, Y. (2016). Relational aggression, Depressive symptoms, And interdependence among school-age children. Personality and Individual Differences, 99, 11-15. doi: 10.1016/j.paid.2016.04.073

Kawabata, Y., Tseng, W. L., \& Crick, N. R. (2014). Adaptive, maladaptive, mediational, and bidirectional processes of relational and physical aggression, relational and physical victimization, and peer liking. Aggressive Behavior, 40(3), 273-287. doi: 10.1002/ ab. 21517

Kessler, R., \& Mroczek, D. (1994). Final version of our non-specific Psychological Distress Scale. Michigan: University of Michigan.

Kushner, S. C., Herzhoff, K., Vrshek-Schallhorn, S., \& Tackett, J. L. (2017). Depression in early adolescence: Contributions from relational aggression and variation in the oxytocin receptor gene. Aggressive Behavior, 44(1), 60-68. doi: 10.1002/ab.21724

Little, R., \& Rubin, D. (2002). Statistical Analysis with Missing Data. Statistical analysis with missing data Second edition. doi: 10.2307/1533221

Little, T., Brauner, J., Jones, S. M., Nock, M. K., \& Hawley, P. H. (2003). Rethinking Aggression: A Typological Examination of the Functions of Aggression. Merrill-Palmer Quarterly, 49(3), 343369. doi: 10.1353/mpq.2003.0014

Little, T. D., Henrich, C. C., Jones, S. M., \& Hawley, P. H. (2003). Disentangling the «whys» from the "whats» of aggressive behaviour. International Journal of Behavioral Development, 27(2), 122133. doi: 10.1080/01650250244000128

Luthar, S. S., \& Ansary, N. S. (2005). Dimensions of adolescent rebellion: risks for academic failure among high- and low-income youth. Development and Psychopathology, 17(1), 231-250. doi: 10.1017/ S0954579405050121

Mariño, M. C., Chaparro, J. J., \& González, C. (1993). Confiabilidad y estructura factorial del CES-D en adolescentes mexicanos. Revista Mexicana de Psicologia, 10, 141-145.
Martínez-Ferrer, B., Murgui-Pérez, S., Musitu, G., \& Monreal-Gimeno, C. (2008). El rol del apoyo parental, las actitudes hacia la escuela y la autoestima en la violencia. International Journal, 8, 679-692. Recuperado de http://redalyc.uaemex. mx/redalyc/pdf/337/33770313.pdf

Meeus, W., Van de Schoot, R., Hawk, S. T., Hale, W. W., \& Branje, S. (2016). Direct Aggression and Generalized Anxiety in Adolescence: Heterogeneity in Development and Intra-Individual Change. Journal of Youth and Adolescence, 45(2), 361-375. doi: 10.1007/ s10964-015-0388-8

Moral, M. V., \& Ovejero, A. (2013). Percepción del clima social familiar y actitudes ante el acoso escolar en adolescentes. European Journal of Investigation in Health, Psychology and Education J. Investig. Health Psychol, 3, 2174-8144. doi: 10.1989/ejihpe.v3i2.32

Moreno, D., Estévez, E., Murgui, S., \& Musitu, G. (2009). Reputación social y violencia relacional en adolescentes: el rol de la soledad, la autoestima y la satisfacción vital. Psicothema, 21(4), 537-542. Recuperado de https://www.uv.es/ lisis/david/ psicothema-reputacion.pdf

Moreno, D., Ramos, M. J., Martínez, B., \& Musitu, G. (2010). Agresión manifiesta y ajuste psicosocial en la adolescencia. SUMMA Psicológica UST, 7(2), 4554. Recuperado de https://doi.org/10.18774/summavol7.num2-122

Musitu, G., Estévez, E., \& Emler, N. (2007). Adjustment problems in the family and school contexts, attitude towards authority, and violent behavior at school in adolescence. Adolescence, 42(168), 779-794. Recuperado de https://bit.ly/2MTzDbJ

Musitu, G., \& García, F. (2004). Consecuencias de la socialización familiar en la cultura española. Psicothema, 16(2), 288-293. Recuperado de http:// www.psicothema.com/pdf/1196.pdf

Pérez-Islas, V., Díaz-Negrete, D. B., \& Fernández-Caceres, C. (2014). Percepción de la violencia social en estudiantes mexicanos de educación media, usuarios y no usuarios de sustancias. Psicología y Salud, 24(2), 255-268. Recuperado de http://revistas.uv.mx/index. php/psicysalud/article/view/929 
Putallaz, M., Grimes, C. L., Foster, K. J., Kupersmidt, J. B., Coie, J. D., \& Dearing, K. (2007). Overt and relational aggression and victimization: Multiple perspectives within the school setting. Journal of School Psychology, 45(5), 523-547. doi: 10.1016/j. jsp.2007.05.003

Roberts, R. E. (1980). Reliability of the CES-D scale in different ethnic contexts. Psychiatry Research, 2(2), 125-134. doi: 10.1016/0165-1781(80)90069-4

Sanchez-Sosa, J. C., Villarreal-Gonzalez, M. E., \& Musitu, G. (2013). Adolescencia y familia:nuevos retos del siglo. Mexico: Trillas.

Sánchez-Sosa, J. C., Villarreal-González, M. E., ÁvilaGuerrero, M. E., Vera-Jiménez, A., \& Musitu, G. (2014). Contextos de socialización y consumo de drogas ilegales en adolescentes escolarizados. Psychosocial Intervention, 23(1), 69-78. doi: 10.50 93/in2014a7

Smilkstein, G., Ashworth, C., \& Montano, D. (1982). Validity and reliability of the family APGAR as a test of family function. The Journal of Family Practice, 15(2), 303-311. Recuperado de https://www.ncbi. nlm.nih.gov/pubmed/7097168

Steffgen, G., Recchia, S., \& Viechtbauer, W. (2013). The link between school climate and violence in school: A meta-analytic review. Aggression and Violent Behavior, 18(2), 300-309. doi: 10.1016/j.avb.2012. 12.001

Trianes, M. V. (2000). La violencia en contextos escolares. Málaga: Aljibe.

Tseng, W. L., Banny, A. M., Kawabata, Y., Crick, N. R., \& Gau, S. S. (2013). A cross-lagged structural equation model of relational aggression, physical aggression, and peer status in a Chinese culture. Aggressive Behavior, 39(4), 301-315. doi: 10.1002/ab.21480
Vaillancourt, T. (2001). Competing for hegemony during adolescence: A link between aggression and social status. (Tesis de doctorado) Recuperada de https:// open.library.ubc.ca/cIRcle/collections/ubctheses/831/ items $/ 1.0053837$

Varela-Garay, R. M., Ávila, M. E., \& Martínez- Ferrer, B. (2013). Violencia escolar: Un análisis desde los diferentes contextos de interacción. Psychosocial Intervention, 22(1), 25-32. doi: 10.5093/in2013a4

Villalta, M. \& Saavedra, E. (2012). Cultura escolar, prácticas de enseñanza y resiliencia en alumnos y profesores de contextos sociales vulnerables. Universitas Psychologica, 11(1), 67-78. Recuperado de http://revistas.javeriana.edu.co/index.php/rev Psycho/article/viewFile/801/1411

Villarreal-González, M. E., Sánchez-Sosa, J. C., Veiga, F. H., \& Moral-Arroyo, G. del. (2011). Contextos de Desarrollo, Malestar Psicológico, Autoestima Social y Violencia Escolar desde una Perspectiva de Género en Adolescentes Mexicanos. Psychosocial Intervention, 20(2), 171-181. doi: 10.5093/in2011v 20n2a5

Voulgaridou, I., \& Kokkinos, C. M. (2015). Relational aggression in adolescents: A review of theoretical and empirical research. Aggression and Violent Behavior, 23, 87-97. doi: 10.1016/j.avb.2015.05.006

Welsh, E., Buchanan, A., Flouri, E., \& Lewis, J. (2004). Involved fathering and child well-being:Fathers' involvement with secondary school age children. New York: Joseph Rowntree Foundation.

Withers, M. C., McWey, L. M., \& Lucier-Greer, M. (2016). Parent-Adolescent Relationship Factors and Adolescent Outcomes Among High-Risk Families. Family Relations, 65(5), 661-672. doi: doi10.1111/ fare. 12220 


\section{Ana Romero-Abrio}

Universidad Pablo de Olavide (España)

Doctoranda en Ciencias Sociales en la Universidad Pablo de Olavide, España (UPO). Líneas de investigación: violencia escolar y violencia filioparental en la adolescencia. Profesora a tiempo parcial en Departamento de Educación y Psicología Social (UPO). Licenciada en Pedagogía por la Universidad de Sevilla (España).

ORCID: 0000-0002-1914-8158

Autor corresponsal: aromabr@upo.es

Gonzalo Musitu Ochoa

Universidad Pablo de Olavide (España)

Catedrático de Psicología Social de la Familia en la Universidad Pablo de Olavide (España). Miembro de número de la Academia de Psicología. Dr. Honoris Causa por la Universidad Autónoma del Estado de Morelos (México). Premio nacional e internacional de investigación. Líneas de investigación: psicología comunitaria, familia y escuela.

gmusoch@upo.es

Juan Evaristo Callejas-Jerónimo

Universidad Pablo de Olavide (España)

Doctor en Ciencias Sociales por la Universidad Pablo de Olavide (España) (UPO). Titulado Superior de Apoyo a la Investigación (UPO). Licenciado en Estadística por la Universidad de Granada (España).

ORCID: 0000-0001-8961-2668

jecaljer@upo.es

\section{Juan Carlos Sánchez-Sosa}

Universidad Autónoma de Nuevo León (México)

Doctor en Psicología por la Universidad Autónoma de Nuevo León, México (UANL). Profesor-Investigador de tiempo completo. Departamento de Investigación y Desarrollo en Psicología (UANL). Miembro del Cuerpo Académico consolidado de Adolescencia y Calidad de Vida. Líneas de investigación: desórdenes alimenticios, adicciones y violencia en adolescencia.

ORCID: 0000-0001-5456-9196

juan.sanchezss@uanl.edu.mx

María Elena Villarreal-González

Universidad Autónoma de Nuevo León (México)

Doctora en Psicología por Universidad Autónoma de Nuevo León, México (UNAL). Profesora-Investigadora tiempo completo. Departamento de Investigación y Desarrollo en Psicología (UANL). Miembro de Red Iberoamericana para el Estudio de la Violencia en la Adolescencia (RIEVA). Líneas de investigación: consumo drogas y violencia en adolescencia.

maria.villarrealgl@uanl.edu.mx 